\title{
Intégrer la notion d'équité : création d'un cadre d'action pour placer la mobilisation du savoir et l'équité en santé au cour des rapports sur l'état de santé des populations
}

\author{
Lesley Ann Dyck, M.A. (1); Susan Snelling, Ph. D. (2); Val Morrison, M.A. (3); Margaret Haworth-Brockman, M. Sc. (4); \\ Donna Atkinson, M.A. (5)
}

Cette synthèse des données probantes a fait l'objet d'une évaluation par les pairs.

Diffuser cet article sur Twitter

\begin{abstract}
Résumé
Les centres de collaboration nationale en santé publique (CCNSP) ont collaboré à l'élaboration d'un cadre d'action visant à intégrer la notion d'équité dans les rapports sur l'état de santé des populations (RESP). Ce cadre d'action rassemble les comptes rendus de recherche et une expérience de terrain fondée sur une approche originale d'apprentissage collaboratif faisant appel aux praticiens en santé publique du Canada.
\end{abstract}

Dans cet article, nous présentons le cadre d'action, nous décrivons le processus d'apprentissage et nous situons les RESP au sein des travaux en cours dans le secteur de la santé publique. Nous analysons ensuite la nature des données probantes sur les déterminants sociaux de la santé à titre de dimension clé pour déterminer sur quoi portent les rapports et comment ces données y sont intégrées. Enfin, nous établissons le lien entre les données et la mise en œuvre en explorant le concept de renseignements exploitables et en détaillant le cadre d'action visant à intégrer la notion d'équité dans les RESP. Nous concluons sur l'importance de placer la mobilisation du savoir au cœur du processus d'élaboration des RESP et nous apportons des suggestions pour les prochaines étapes. Notre objectif est d'encourager les praticiens à utiliser le cadre d'action, à en discuter et, ultimement, à le renforcer.

Mots-clés : rapports sur l'état de santé des populations, équité en santé, iniquité, déterminants sociaux de la santé, mobilisation du savoir

\section{Introduction}

La description des différences relatives à l'état de santé entre les populations ou les groupes et au sein de ceux-ci joue un rôle central dans les rapports sur l'état de santé des populations (RESP) au Canada ${ }^{1}$. Nous nous intéressons particulièrement ici aux différences relatives à l'état de santé qu'on peut considérer comme systématiques, injustes et évitables. Ces différences sont souvent décrites comme étant des inégalités (ou iniquités) sociales et sont attribuables aux relations de pouvoir et aux structures inégalitaires dans les sociétés ${ }^{2,3}$. Afin d'éliminer les iniquités et d'améliorer l'équité en santé, nous devons prendre des mesures concertées pour modifier les déterminants sociaux responsables des disparités en santé, qui incluent un large éventail de facteurs sociaux, politiques et économiques ${ }^{4}$. C'est ce qui est au cœur de l'intégration de la notion d'équité au processus d'élaboration des RESP.

Le secteur de la santé publique a plusieurs rôles à jouer dans la modification des déterminants sociaux de la santé et l'amélioration de l'équité en santés. Le rôle auquel nous nous intéressons ici est celui de l'évaluation et des rapports. La production de rapports centrés sur les différences en matière de santé entre les divers groupes socioéconomiques plutôt que sur l'ajustement
Points saillants

- Les RESP sont au cœur de la pratique en santé publique, mais, au Canada, ils ne décrivent généralement pas explicitement les iniquités en santé et ne renferment pas de recommandations sur les mesures à prendre pour faire progresser l'équité en santé.

- Le projet, fondé sur une approche originale d'apprentissage collaboratif, visait à déterminer comment mieux intégrer la notion d'équité en santé dans les processus d'élaboration des RESP.

- Le résultat du projet est un cadre d'action qui place la mobilisation du savoir en première place afin de soutenir la mise en œuvre d'un processus d'élaboration des RESP qui encouragera la prise de mesures visant à améliorer l'équité en santé.

pour tenir compte de ces différences s'est avérée être une pratique prometteuse pour faire progresser l'équité en santé ${ }^{\text {. }}$ Mentionner sciemment les iniquités en santé touche à la fois la fonction fondamentale de surveillance de la santé publique et les modalités usuelles de rédaction des RESP. On a fait valoir que, lorsque sont évaluées et prises en compte les iniquités en santé, notamment par la mention de stratégies efficaces pour les réduire, les organismes de santé publique sont davantage susceptibles d'agir et de se soutenir mutuellement afin de réduire ensemble ces iniquités ${ }^{7}$.

Rattachement des auteurs :

1. Centre de collaboration nationale des déterminants de la santé, Université St. Francis Xavier, Antigonish (Nouvelle-Écosse), Canada

2. Centre de collaboration nationale des méthodes et outils, Université McMaster, Hamilton (Ontario), Canada

3. Centre de collaboration nationale sur les politiques publiques et la santé, Institut national de santé publique du Québec, Montréal (Québec), Canada

4. Centre de collaboration nationale des maladies infectieuses, Université du Manitoba, Winnipeg (Manitoba), Canada

5. Centre de collaboration nationale de la santé autochtone, Université du Nord de la Colombie-Britannique, Prince George (Colombie-Britannique), Canada

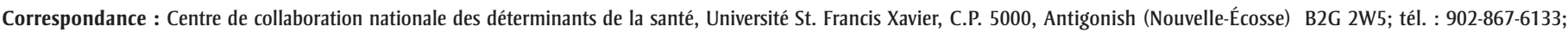
courriel : nccdh@stfx.ca 
Nous avons recherché les RESP au Canada faisant état de l'intégration efficace des questions d'équité en santé et des déterminants sociaux de la santé et nous avons constaté qu'ils étaient peu nombreux. Dans ceux que nous avons trouvés, aucune approche uniforme ou normalisée ne semblait avoir été utilisée $e^{8-11}$. Cela nous a amené à nous poser plusieurs questions. À quoi ressemble l'intégration efficace de l'équité en santé dans un processus d'élaboration des RESP? À quoi devonsnous prêter attention pour réaliser cette intégration avec succès? En quoi ce processus contribue-t-il à un passage à l'action en matière de déterminants sociaux de la santé qui fasse progresser l'équité en santé? Tout en explorant ces questions, nous avons élaboré un document intitulé L'intégration de la notion d'équité dans les rapports sur l'état de santé des populations : un cadre d'action $^{12}$, un cadre d'action destiné à l'élaboration des RESP et susceptible, selon nous, de soutenir les organismes en santé publique dans leur travail d'évaluation et de rapports en vue de favoriser le passage à l'action en matière de déterminants sociaux de la santé et d'équité en santé.

Dans cet article, nous présentons le cadre d'action et expliquons le contexte de son élaboration. Nous décrivons d'abord brièvement notre processus d'apprentissage, puis nous situons les RESP au sein des travaux en santé publique. Nous analysons ensuite la nature des données probantes sur les déterminants sociaux de la santé à titre de dimension clé pour déterminer sur quoi portent les rapports et comment ces données y sont intégrées. Enfin, nous créons un lien entre l'information et la mise en œuvre en explorant la notion de données exploitables et nous présentons le cadre d'action visant à intégrer la notion d'équité dans les RESP. Nous concluons l'article sur l'importance de placer la mobilisation du savoir au cœur du processus d'élaboration des RESP et nous offrons des suggestions pour les prochaines étapes.

\section{Méthodologie : le processus d'élaboration de notre cadre d'action}

Notre processus d'apprentissage a été conduit par le Centre de collaboration nationale des déterminants de la santé (CCNDS), l'un des six centres de collaboration nationale en santé publique créés en 2005 afin de renforcer l'application et la transmission des connaissances en santé publique au Canada ${ }^{13}$. Le CCNDS a mis sur pied un cercle d'apprentissage composé de spécialistes de l'équité en santé de l'ensemble du Canada, provenant de dix organismes en santé publique - amenant ainsi une variété de points de vue (gestionnaires de programme, médecins hygiénistes, analystes des politiques et d'épidémiologistes œuvrant dans des unités de santé, des autorités régionales de la santé ou des autorités provinciales de la santé publique, etc.) - ainsi que d'universités (chercheurs). Leur tâche consistait à déterminer et à explorer les enjeux fondamentaux associés à l'intégration de l'équité en santé dans les RESP et à recenser les pratiques prometteuses en contexte canadien. Leurs travaux se sont traduits par la publication de la série Apprenons ensemble ${ }^{14}$, une collection de documents décrivant le processus employé par le cercle d'apprentissage et les questions clés étudiées lors de chaque rencontre du cercle. De ce projet est née une collaboration avec les cinq autres centres de collaboration nationale en santé publique visant à élaborer L'intégration de la notion d'équité dans les rapports sur l'état de santé des populations : un cadre d'action ${ }^{12}$. Ce cadre d'action a été conçu et peaufiné à l'aide d'entrevues auprès de dix intervenants importants à l'échelle locale, provinciale et nationale au Canada. Différentes versions du document ont également été présentées et examinées dans le cadre d'ateliers lors de trois conférences sur la santé publique au Canada* et d'un webinaire ${ }^{\dagger}$. Les commentaires de plus de 100 praticiens en santé publique qui ont participé à ces événements ont été recueillis par le biais des notes sur les délibérations et dans les évaluations et ont été intégrés lors de la rédaction de la version finale du cadre d'action.

\section{Résultats : les composantes de production d'un rapport sur l'état de santé des populations tenant compte de l'équité}

\section{Qu'est-ce qu'un rapport sur l'état de santé d'une population?}

Les six fonctions essentielles des autorités sanitaires canadiennes sont les suivantes : évaluation de la santé de la population, promotion de la santé, prévention et contrôle des maladies et des blessures, protection de la santé, surveillance et enfin préparation en cas d'urgence et intervention en cas d'épidémie ${ }^{15,16}$. Ces fonctions sont exercées, totalement ou partiellement, à toutes les échelles de gouvernement (fédéral, provincial, territorial et leurs autorités déléguées, dont les autorités régionales de la santé). Tous les gouvernements nomment un administrateur en chef de la santé publique ou un médecin hygiéniste qui dirige les interventions en matière de santé publique dans son administration $^{15}$, la législation et les responsabilités variant quelque peu d'une province ou d'un territoire à l'autre.

Rédiger un rapport sur l'état de santé ne fait pas partie des fonctions essentielles mais constitue un outil de base pour remplir les fonctions essentielles du mandat de la santé publique. Dans un résumé du profil structurel de la santé publique au Canada, le Centre de collaboration nationale sur les politiques publiques et la santé a constaté que le mandat consistant à rendre compte de l'évaluation et de la surveillance de la santé de la population (soit les deux fonctions clés les plus pertinentes pour les RESP) n'était pas rempli de la même façon dans les différentes administrations. Ainsi, à l'échelle fédérale, « [d]ans les six mois suivant la fin de chaque exercice, l'administrateur en chef présente au ministre un rapport sur l'état de santé publique au Canada ${ }^{17}$. Un exemple de ce qui existe à l'échelle provinciale est illustré par la situation en Colombie-Britannique, où, selon la Public Health Act de la province, l'évaluation de la santé de la population relève principalement du médecin-hygiéniste provincial. À l'échelle régionale, au Manitoba, par exemple, l'évaluation de la santé de la population en tant que fonction de la santé publique est partiellement réalisée par les autorités régionales, certaines de ces composantes étant prévues dans la Loi sur les offices régionaux de la santé ${ }^{17}$.

\section{L'intégration de la notion d'équité}

Dans le cadre de notre projet, on a défini comme RESP intégrant la notion d'équité tout « instrument que l'on peut fonder sur les connaissances scientifiques et locales existantes de façon à éclairer les décisions, améliorer les programmes de santé et

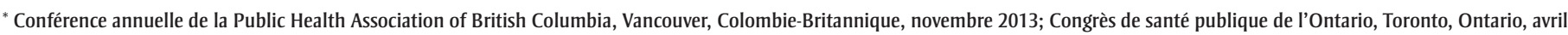
2014; Conférence annuelle de l'Association canadienne de santé publique (ACSP), Toronto, Ontario, mai 2014.

† Organisé par CHNET-Works!, mars 2014. 
réduire les iniquités en santé $»^{1, p .}{ }^{2}$. Les RESP intègrent à la fois des données de surveillance et d'autres données et sont généralement utilisés pour souligner des enjeux ou des sujets de santé publique précis $^{1}$. Il demeure que l'une des difficultés à un examen de l'équité en santé dans le contexte des RESP est l'absence de format, de contenu ou de processus normalisés pour ces rapports. Si nous considérons à une échelle plus large les RESP comme une catégorie d'évaluation de la santé de la population, nous pouvons les inclure aux connaissances en santé (figure 1$)^{18}$. Un RESP peut être ainsi vu comme un produit (document imprimé, fichier électronique ou page Web) qui fournit une évaluation de la santé de la population et génère des connaissances exploitables en matière de santé publique. Il est fondé sur les sources de données qu'on utilise en surveillance comme en recherche en santé publique (figure 1) ${ }^{18}$.

\section{Caractérisation du processus d'évaluation et de ses objectifs}

L'obtention d'information sur la manière de créer un processus d'élaboration efficace et utile des rapports ou évaluations sur l'état de santé des populations est entravée par le manque de lignes directrices reconnues en matière de rapports et de processus. L'évaluation de la santé communautaire est une approche globale de développement de la collectivité qui fait normalement partie d'un processus plus étendu d'amélioration de la santé de la collectivité $^{19}$. Cette évaluation est souvent menée par des organismes communautaires en partenariat avec le secteur de la santé, la plupart du temps aux États-Unis ${ }^{20}$. Comme toute évaluation en santé communautaire, les RESP constituent à la fois l'activité de détermination et de priorisation des problèmes de santé de la population et son résultat, et ils varient en fonction de la taille et la nature de la collectivité, l'organisme ou les partenaires qui en sont à l'origine ainsi que leurs objectifs, les ressources disponibles et d'autres facteurs locaux $^{20}$. Le processus d'élaboration des RESP étant mené au Canada par le secteur de la santé publique, il est susceptible de produire des renseignements exploitables davantage pour le secteur de la santé publique que pour l'ensemble de la collectivité. Comme nous allons le voir, il devient alors

FIGURE 1

\section{Surveillance en santé publique dans le contexte plus large des connaissances en santé}

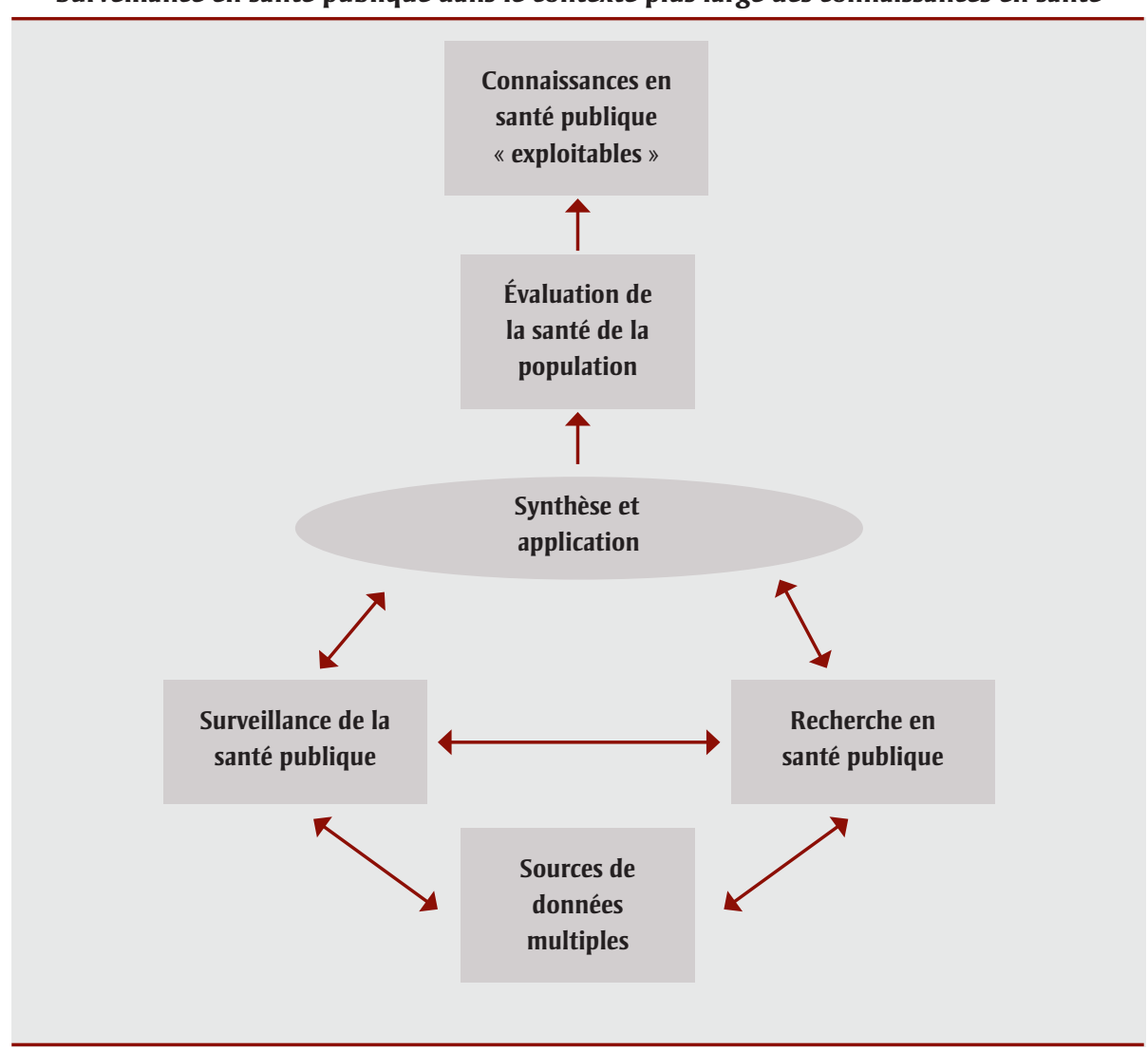

Source : Lexicon, definitions, and conceptual framework for public health surveillance ${ }^{18, p .13}$. difficile de faire état des iniquités en santé en vue d'encourager l'action visant à améliorer les déterminants sociaux de la santé.

En examinant les rapports sur la santé publique publiés au Canada, nous avons constaté qu'un rapport pouvait avoir un ou plusieurs objectifs, selon le contexte ou le sujet : a) améliorer la responsabilisation et évaluer la qualité ou l'efficacité d'un programme ou d'un service lorsque l'accent est mis sur les programmes et services, b) analyser les changements dans l'état de santé d'une population au cours d'une période ou dans diverses zones géographiques lorsque l'accent est mis sur la population et c) déterminer et quantifier les disparités en santé afin d'établir les différences entre divers groupes lorsque l'accent est mis sur les disparités en santé21. Nous en avons conclu que les RESP étant fondés sur des données probantes et de nature publique - quoique non normalisés ou ne traitant pas nécessairement des enjeux en matière d'équité -, ils ont pu devenir un élément clé de l'élaboration et du réalignement des politiques et des programmes en santé publique ${ }^{1}$.

Un second auditoire est souvent visé par ces rapports, habituellement par la mention d'exemples d'interventions intersectorielles et de recommandations d'action : celui des intervenants en matière de politiques publiques saines agissant hors du secteur de la santé publique (autres ministères du gouvernement, municipalités et organismes communautaires ${ }^{21}$. En matière d'actions visant à améliorer les déterminants sociaux de la santé, ce second auditoire « externe » est essentiel. En effet, l'équité en santé est déterminée par un éventail plus large de facteurs sociaux associés aux politiques, normes et valeurs dont la plupart échappent à l'influence du secteur de la santé. Si les données servent à agir uniquement en santé publique, on ne pourra lutter efficacement contre les iniquités systématiques en santé.

Notre cercle d'apprentissage composé de praticiens et de chercheurs en santé publique en est arrivé à la conclusion qu'un rapport réussi est un rapport qui est utilisé. Pour que les données contenues dans les RESP soient exploitables, il est essentiel de déterminer la meilleure façon d'y intégrer l'équité en santé. Les praticiens en santé publique des diverses régions du Canada nous ont appris que l'utilisation réussie d'un rapport repose sur son format 
et son contenu ainsi que sur la manière dont les intervenants ont été mobilisés dans la collectivité pour la collecte de données et le processus d'élaboration.

Avant d'examiner plus en détail la mobilisation en tant que principe clé des RESP, nous allons définir ce que sont des données probantes valides pour l'élaboration d'un RESP dans une perspective d'équité en santé.

\section{Les données probantes relatives à l'« iniquité en santé », enjeu de santé publique conduisant à l'action}

Les données probantes relatives à l'iniquité en santé en tant qu'enjeu de santé publique conduisant à l'action sont en croissance, en grande partie grâce à la mise sur pied de la Commission des déterminants sociaux de la santé (CDSS) de l'Organisation mondiale de la santé (OMS). L'un des réseaux d'expertise de la CDSS a créé un guide sur la construction de données probantes en matière de déterminants sociaux de la santé en mettant en évidence six problèmes conceptuels et théoriques ${ }^{22}$. L'une des principales constatations du réseau en matière d'application des connaissances sur l'iniquité est que les données probantes à elles seules ne sont pas synonymes de succès ni ne poussent à agir. On doit raffiner les données et mobiliser tous les intervenants qui participent à la collecte de données, à la transformation de ces dernières en politiques et à la transformation des politiques en action et mesures concrètes. En conclusion de ce guide, on reconnait que, bien que nous en sachions beaucoup sur les facteurs sociaux qui ont une influence sur la santé, nos connaissances ne sont pas applicables de façon universelle. Elles « [...] doivent donc être interprétées avec une perspective qui tient compte de leur prépondérance, leur signification et leur pertinence dans des contextes locaux particuliers » [traduction] ${ }^{22, \text { p. } 218}$. Ce constat met en lumière l'importance de mobiliser les intervenants qui connaissent le contexte local pour la collecte, l'analyse et la déclaration des données sur l'état de santé de la population si on veut intégrer efficacement les considérations relatives à l'équité en santé.

De nombreux RESP au Canada ont abordé les défis conceptuels de diverses manières afin de réellement intégrer une perspective d'équité en santée-11,23-26. Ces rapports ont en commun, d'une part, d'être explicites quant à l'accent mis sur l'équité et sur leur intention de susciter une action pour améliorer l'équité en santé et, d'autre part, de faire état des collaborations et consultations avec les organismes et citoyens qui se sont révélés indispensables à leur production. Toutefois, ces rapports n'ont pas d'approche normalisée et la plupart ne paraissent qu'une fois, ce qui rend difficile le repérage des tendances et l'évaluation de leur impact collectif sur la diminution des iniquités en santé. Font exception à cette règle de parution unique les rapports Unequal city de Toronto parus en 2010 et $2015^{11}$ et les évaluations de la santé communautaire parues en 2004, 2009 et 2015 à Brandon $^{23}$ et en 2004, 2009-2010 et 2015 à Winnipeg ${ }^{26}$.

Une partie des difficultés à repérer les tendances provient de la diversité des mesures et indicateurs utilisés pour évaluer et surveiller l'équité en santé. Ces difficultés ont suscité un intérêt particulier depuis environ dix ans au Canada, ce qui a mené à la création concertée de processus d'élaboration d'indicateurs d'équitée ${ }^{27,28}$, à l'élaboration et à l'application de divers indices de privation socioéconomique ${ }^{29-31}$ et à la rédaction d'un rapport sur l'évolution des indicateurs liés à l'équité ${ }^{32}$. Les épidémiologistes poursuivent leurs discussions sur les meilleures méthodes de mesure et de repérage de l'équité et des iniquités en santés3-36, mais certains avancent que ce n'est pas tant la qualité des mesures qui pose problème que l'obtention d'un consensus en ce qui a trait aux indicateurs à utiliser et la promotion d'une collecte et d'une déclaration de données constantes au fil du temps ${ }^{37,38}$.

Divers problèmes conceptuels et méthodologiques importants faisant obstacle à l'accessibilité à des données pertinentes de grande qualité ne sont pas résolus. Par exemple, les données administratives sur la santé ne contiennent habituellement pas d'information sur le revenu, l'origine ethnique, l'emploi et le niveau de scolarité, information qui nous permettrait de répartir les données de population de manière à favoriser l'évaluation de l'équité en santé. Il est donc très difficile d'observer les différences dans l'état de santé entre populations, en particulier les tendances pour les groupes depuis longtemps marginalisés ou opprimés (p. ex. les personnes handicapées et les membres de la communauté LGBTA). De même, les données sur la santé des Autochtones au Canada, aux États-Unis, en Nouvelle-Zélande et en
Australie sont insuffisantes ou de piètre qualité. Ce n'est que récemment que les enquêtes sur la santé au Canada et ailleurs ont offert la possibilité aux Autochtones de s'identifier comme tels, permettant ainsi aux analystes de mieux comprendre les iniquités en santé touchant les Autochtones vivant hors réserve et en milieu urbain. Enfin, les relations de cause à effet entre les interventions et leurs répercussions sur les iniquités en santé demeurent mal comprises $^{22}$, rendant difficile le choix des données à recueillir et les modalités de cueillette dans le cadre des évaluations courantes de programme. Toutes ces contraintes ralentissent « la mise en œuvre stratégique d'interventions en santé publique fondées sur des données probantes et visant à prévenir la mortalité évitable » [traduction] ${ }^{39, p .644}$.

Au Canada, les données relatives aux populations des Premières Nations, des Inuits et des Métis sont souvent inexistantes, incomplètes, inadaptées sur le plan culturel et affectées par les enjeux fondamentaux de pouvoir et de contrôle, notamment les contestations de compétences associées aux différents ordres de gouvernement ${ }^{40,41}$. Des tentatives ont eu lieu pour surmonter ces difficultés, notamment le travail du Centre de gouvernance de l'information des Premières Nations (CGIPN). Ce dernier s'est attaché à placer les communautés au cœur du processus d'élaboration des RESP en mettant sur pied l'Enquête régionale sur la santé des Premières Nations. Cette initiative a permis aux communautés d'acquérir un certain contrôle sur le processus d'élaboration des RESP, notamment sur les décisions à propos de la participation, sur le choix des indicateurs, sur la propriété des données et sur les renseignements fournis ${ }^{42}$. Toutefois, il ne s'agit que d'une première étape, étant donné que l'Enquête régionale sur la santé des Premières Nations ne tient compte ni du grand nombre d'Autochtones vivant en milieu urbain au Canada ni des autres groupes autochtones (p. ex. les Métis).

\section{Mobilisation et information exploitable sur la santé}

Corburn et Cohen font valoir que " la rédaction, la mesure, le suivi et la déclaration des indicateurs ne devraient pas être perçus comme un processus technique s'adressant uniquement aux experts, mais plutôt comme une occasion d'élaborer des politiques scientifiques participatives, ce 
que nous appelons la gouvernance " [traduction] ${ }^{38, p .2}$. Pour eux, la gouvernance concerne non seulement les institutions en tant que telles, mais également les " normes, routines et pratiques » visant la définition des enjeux de la recherche et des politiques, les données probantes utilisées et les acteurs sociaux dont l'expertise est jugée suffisante pour qu'ils puissent participer aux décisions. Ce sont donc l'élaboration et l'utilisation des indicateurs d'équité ou d'iniquités en santé qui ouvrent à une gouvernance saine et équitable ${ }^{38}$. C'est là-dessus qu'insiste le rapport européen de l'OMS sur la gouvernance pour l'équité en santé ${ }^{43}$ : on y recommande de faire de l'équité et de l'équité en santé des marqueurs essentiels pour une société équitable et durable, et on exige que les données probantes et l'analyse soient liées aux objectifs sectoriels généraux et que les différents secteurs et intervenants utilisent les mêmes méthodes d'évaluation.

Pour les établissements en santé publique, disposer d'indicateurs destinés à façonner les politiques et encourager l'action visant à améliorer l'équité en santé implique de pouvoir aller au-delà des indicateurs traditionnels et de mobiliser un large éventail d'intervenants par de nouveaux moyens. Il est important de reconnaître que

[...] les indicateurs traditionnels qui mesurent la morbidité et la mortalité attribuent généralement l'amélioration de la santé à la communauté médicale, au milieu de la santé publique ou à des institutions désignées de manière imprécise comme l'économie, l'éducation ou l'environnement bâti. Il en résulte est une trop grande insistance sur les solutions relevant de la médecine ou de la santé publique alors que personne ne semble en mesure de définir précisément les institutions et les politiques qui devraient changer afin de promouvoir une meilleure équité en santé. [traduction] $]^{38, \text { p. }} 5$

Concevoir des RESP fondés sur la mobilisation de la collectivité est essentiel pour intégrer l'équité en santé de manière à la fois à orienter la création et la prestation de programmes et de services en santé publique et à favoriser la prise de mesures intersectorielles pour lutter contre l'iniquité en santé. L'adoption d'une telle approche exige que le secteur de la santé publique aille au-delà de la surveillance et du suivi traditionnels et ne se limite pas à une définition de l'état de santé d'une population reposant sur l'agrégation de microdonnées sur la santé. Étant donné que les données probantes sont toujours teintées de valeurs, si nous n'appliquons pas une perspective d'équité à leur collecte, leur analyse et leur synthèse, nous risquons de négliger les problèmes de pouvoir et d'oppression systématiques potentiellement liés aux mesures de l'état de santé d'une population.

En adoptant une approche fondée sur la mobilisation de la collectivité pour assumer son rôle d'évaluation et de rapports, le secteur de la santé publique peut utiliser la puissance des RESP pour fusionner données probantes et valeurs - ici, les valeurs d'équité et d'impartialité. Cette approche d'élaboration des RESP rend visibles ces valeurs dans les données probantes et accroît la possibilité que ces dernières soient exploitables. On a défini dans le cadre d'action trois composantes essentielles soutenant l'intégration de la notion d'équité dans les RESP : la communication, la collaboration et l'application d'une perspective liée aux valeurs d'équité en santé ${ }^{12}$ (voir l'« axe de mobilisation du savoir » sur la figure 2).

\section{Analyse : un cadre d'action pour les rapports sur l'état de santé des populations}

Dans les RESP classiques, le processus de transfert des connaissances en action met l'accent sur les données probantes et se termine par un résumé de l'état de santé. Dans un processus d'élaboration de rapports visant l'action, la mobilisation du savoir passe par la combinaison des connaissances issues de la recherche et d'autres types de connaissances et implique leur transformation en recommandations stratégiques visant le passage à l'action. Cette approche orientée vers l'action, quoique peu répandue dans les RESP, prend de plus en plus d'importance au Canada $a^{9,11,24}$.

\section{Une orientation vers l'action}

Nous proposons un cadre d'action pour les RESP qui soit orienté vers l'action, qui place la mobilisation du savoir inspiré par l'équité au cœur du processus et qui s'appuie sur les étapes d'élaboration des rapports en santé des populations, comme l'illustre la figure 2. Même si on souhaite comme résultat à long terme l'amélioration de l'équité dans l'état de santé des populations, ce cadre d'action est original au sens où il vise d'abord concrètement à ce que « la collectivité [soit] mieux outillée pour atténuer les problèmes d'équité en santé12,p.9 ", plaçant ainsi le leadership intersectoriel local au cœur même du processus. Sont également définis les rôles et les attentes spécifiques liés à chacun des trois groupes d'intervenants principaux engagés dans le processus, à savoir le secteur de la santé publique, les partenaires communautaires et les chercheurs.

Ce cadre d'action s'inspire de deux cadres d'action similaires fondés sur des données probantes : le Modèle de prise de décision en santé publique fondée sur les données probantes du Centre de collaboration nationale des méthodes et outils ${ }^{44}$ et l'Action Cycle élaboré par la Robert Wood Johnson Foundation $^{45}$. Nous présentons ici une courte synthèse de ce cadre d'action, décrit par ailleurs en détail avec des exemples de pratiques prometteuses dans L'intégration de la notion d'équité dans les rapports sur l'état de santé des populations : un cadre d'action ${ }^{12}$, un document accessible sur le site Internet du CCNDS.

\section{La dimension de la mobilisation du savoir}

Dans le cadre d'action que nous proposons, la mobilisation du savoir est la pierre angulaire de la synthèse, du transfert et de la communication des connaissances indispensables au processus d'élaboration des RESP. Elle est adaptée aux utilisateurs visés par le cadre d'action (leadership communautaire intersectoriel) et repose sur une approche collaborative qui intègre au processus la notion d'équité. Elle est composée de trois éléments principaux, qui se rattachent à où, qui et comment (encadré 1). Dans le document décrivant l'ensemble du cadre d'action $^{12}$ sont fournis des exemples concrets, au Canada et à l'étranger, d'une intense mobilisation du savoir pour l'élaboration de RESP intégrant la notion d'équité. Parmi ces exemples figurent aussi bien des rapports qui appliquent une perspective explicite d'équité en santé que des rapports qui fournissent de bons exemples de collaboration et de communication autour du sujet de l'équité en santé et des RESP.

\section{Les étapes de l'élaboration et de la mise en cuvre des rapports}

Dans le cadre d'action, sept étapes sont nécessaires pour l'élaboration et la mise en œuvre des RESP. À chaque étape, des questions clés visent à orienter les activités afin 


\section{FIGURE 2 \\ L'intégration de la notion d'équité dans les rapports sur l'état de santé des populations : un cadre d'action}

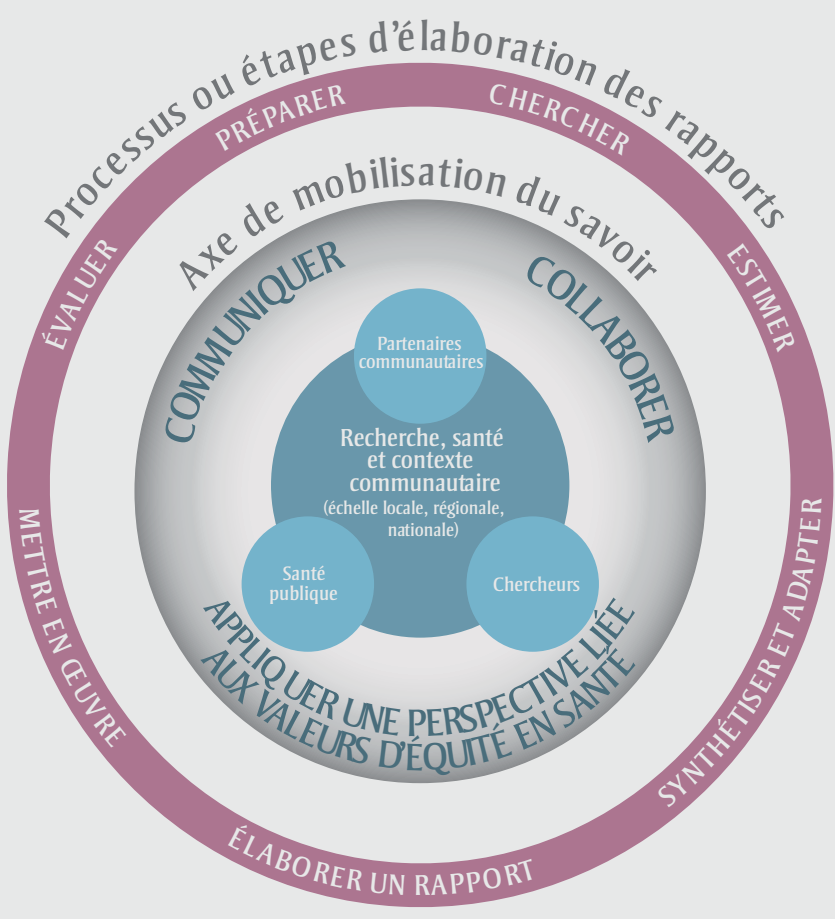

A. AXE DE MOBILISATION DU SAVOIR

Où - procure le contexte (recherche, santé et contexte communautaire)

Qui-décrit les principaux acteurs (santé publique, chercheurs, partenaires communautaires)

Comment - décrit l'approche à suivre tout au long du processus communiquer, collaborer, appliquer une perspective liée aux valeurs d'équité en santé)
B. PROCESSUS OU ÉTAPES D'ÉLABORATION DES RAPPORTS

Quoi - décrit les étapes du processus d'élaboration de RESP et les principales questions à considérer pour intégrer la notion dequité en santé (prepare, chercher, adapter, elaborer un rapport, mettre en œuvre, évaluer)

Source : Sommaire - Élaboration de rapports sur l'état de santé des populations en tenant compte de l'équité : cadre d'action ${ }^{49, p .2}$

de mettre en place les bonnes structures pour soutenir le travail d'élaboration des RESP intégrant la notion d'équité (encadré 2 ). Nous avons également retenu, comme nous l'avons fait pour la mobilisation du savoir, un certain nombre de pratiques prometteuses associées à une ou plusieurs étapes du processus d'élaboration des rapports. Ces pratiques figurent aussi dans le document décrivant le cadre d'action ${ }^{12}$.
Notons que la seule étape du processus pour laquelle nous n'avons pas réussi à trouver de pratique prometteuse est l'étape de l'évaluation. L'un des défis autour de l'évaluation des résultats, par exemple les répercussions des changements apportés aux politiques, est la nature à long terme du processus. Comme l'a souligné Hilary Graham, cette étape a un impact sur l'engagement politique à l'égard d'une meilleure équité en santé, qui « [...] risque de diminuer rapidement, surtout si les changements dans les politiques [...] se révèlent insuffisants pour assurer une réduction des inégalités [...] dans les courtes périodes que les gouvernements fixent habituellement pour l'atteinte de leurs objectifs stratégiques »[traduction] $]^{46, p .475}$. Notre processus de consultation nous a appris que certains de nos informateurs évaluent ou prévoient d'évaluer leurs activités relatives en lien avec les RESP, mais nous n'avons pas réussi à obtenir d'exemples concrets. Dans une prochaine étape de conception du cadre d'action axé sur l'équité pour l'élaboration des RESP, il sera important de recenser toutes les évaluations réalisées et d'en tirer des leçons.

\section{Conclusion : contribution et poursuite de l'élaboration du cadre d'action}

Comme nous l'ont mentionné les membres de notre cercle d'apprentissage ainsi que d'autres informateurs en santé publique, un rapport non utilisé n'aidera pas à faire avancer l'équité en santé ${ }^{21}$. La mobilisation du savoir constitue donc une caractéristique centrale d'un cadre d'action axé sur l'équité pour l'élaboration des RESP. Un

\section{ENCADRÉ 1 \\ Dimension de mobilisation du savoir}

Où - Le processus d'élaboration de RESP peut se faire à l'échelle locale, régionale ou nationale. À chaque échelon, les personnes impliquées, les organismes, les cultures politiques et les données diffèrent. En dernière instance, le contexte communautaire et les enjeux locaux orientent le processus d'élaboration des rapports et, inversement, sont influencés par lui dans un contexte systémique plus global. À long terme, la collectivité dans son ensemble est mieux outillée pour atténuer les problèmes d'équité en santé, ce qui permet d'améliorer l'équité en santé en contexte local.

Qui - Les personnes œuvrant dans le secteur de la santé publique, les partenaires communautaires et les chercheurs sont les principaux acteurs du processus d'élaboration des RESP. Soulignnons qu'un processus dirigé par un seul type d'acteurs amènera moins souvent à une action. La capacité de tous à faire preuve de leadership et à agir est fondamentale dans l'intégration efficace de l'équité en santé au sein du processus. Le secteur de la santé publique est essentiel dans l'élaboration de RESP, les acteurs et les défenseurs de la santé publique étant bien placés pour fournir le leadership nécessaire pour assurer l'efficacité du processus d'élaboration des RESP. Les partenaires communautaires (administration locale, organismes communautaires et autres responsables communautaires) ont néanmoins une responsabilité tout au long du processus et l'apport des chercheurs, qui œuvrent dans des milieux et des sphères d'activité différents, se révèle essentiel à diverses étapes du processus.

Comment - Il n'y a pas de « modèle universel » de mobilisation du savoir nécessaire à l'élaboration des RESP. Cela dit, il importe tout de même de toujours respecter certains principes de base, mentionnés dans le cadre d'action sous forme de questions à considérer réparties en trois catégories : a) appliquer une perspective liée aux valeurs d'équité en santé, b) collaborer et c) communiquer.

Source : Texte adapté de Sommaire - Élaboration de rapports sur l'état de santé des populations en tenant compte de l'équité : cadre d'action ${ }^{49, p .3}$. 


\section{ENCADRÉ 2 \\ Questions à se poser à chacune des sept étapes du processus d'élaboration de RESP intégrant la notion d'équité}

1. Préparer - Qui doit faire partie du processus? Quels sont les questions-clés et les enjeux ou problèmes principaux? Comment les valeurs d'équité sont-elles intégrées à nos questions d'enquête?

2. Chercher - Quel est le meilleur moyen de trouver des données probantes issues de la recherche qui soient pertinentes? Quels indicateurs nous permettront de répondre à notre question de recherche? Quelles sont les autres données disponibles? Est-il utile d'envisager de recueillir des données supplémentaires?

3. Estimer - Quelles sont les sources de données et quel est le niveau de qualité des données? Quelles sont les limites de ces sources et de ces données? Peut-on utiliser les données probantes issues d'autres travaux de recherche quantitative, qualitative ou participative pour compléter nos données? Comment les méthodes de recherche, la collecte et l'analyse des données intègrent-elles les valeurs d'équité en santé? Les divers indicateurs mesurent-ils à la fois les points forts et les points faibles? Dans quelle mesure les données de population sont-elles disponibles en fonction des caractéristiques géographiques, sociales et économiques?

4. Synthétiser et adapter - Comment synthétiser, adapter et intégrer différents types de données probantes afin de brosser un tableau plus complet des iniquités? Quelles recommandations pouvons-nous formuler pour soutenir une pratique fondée sur les données probantes? Comment les valeurs d'équité en santé y sont-elles intégrées? En quoi les recommandations s'appliquent-elles au contexte local?

5. Élaborer un rapport - Quel est notre public-cible et quel est le meilleur moyen de lui transmettre ce que nous savons?

6. Mettre en œuvre - Comment pouvons-nous organiser les résultats de manière à mobiliser chacun? Quel est le meilleur moyen d'envisager l'éventail des actions potentielles, qui va de la mobilisation des membres de la collectivité à l'élaboration de politiques? Comment pouvons-nous travailler en collaboration afin de mettre en œuvre ces actions potentielles?

7. Évaluer - Dans quelle mesure le processus d'élaboration de RESP a-t-il contribué à l'atteinte de nos objectifs organisationnels de départ en matière de rapport, parmi lesquels figurent l'inclusion et l'intégration de l'amélioration de l'équité? Dans quelle mesure ce processus a-t-il amélioré la capacité de la collectivité à prendre des mesures concernant les déterminants sociaux de la santé et l'équité en santé?

Source : Texte adapté du Cadre d'action axé sur l'équité pour l'élaboration de rapports sur l'état de santé des populations ${ }^{12, p .35}$.

processus d'élaboration des RESP intégrant la notion d'équité doit être conçu autour d'un processus itératif adaptable au contexte et à la capacité des intervenants et capable de puiser dans les pratiques prometteuses d'autres disciplines et d'autres autorités. Nos discussions avec un grand nombre de praticiens en santé publique nous ont également appris que les bons RESP intégrant la notion d'équité sont transparents quant à la manière dont sont reliées données probantes et valeurs associées à la justice sociale. Il est alors davantage probable que les données soient utilisées pour orienter d'autres processus de plus grande ampleur, notamment l'évaluation et l'amélioration de la santé communautaire, les initiatives de lutte la recherche tournée vers la pratique dans ces domaines :

1. une estimation des processus actuels d'élaboration des RESP mis en œuvre dans le secteur de la santé publique au Canada, en vue d'évaluer à la fois les processus et les résultats, notamment les changements apportés aux politiques $^{47}$;

2. la conception de lignes directrices claires pour la production de RESP qui intègrent de manière efficace la notion d'équité en santé ainsi que des objectifs en matière de politiques organisationnelles et de politiques publiques saines $^{48}$.

Nous espérons que ce cadre d'action contribuera à la création et à l'amélioration de rapports sur l'état de santé des populations aptes à faire progresser l'équité en santé au Canada. Nous avons hâte d'apprendre comment les organismes en santé publique vont utiliser leurs processus d'élaboration des RESP pour améliorer l'équité en santé dans leurs collectivités.

\section{Remerciements}

Ce projet a été financé par le budget de fonctionnement du Centre de collaboration nationale des déterminants de la santé, qui est financé par l'Agence de la santé publique du Canada. Nous aimerions remercier tout particulièrement Tannis Cheadle pour son aide dans l'élaboration et la rédaction du document décrivant le cadre d'action. Nous tenons aussi à remercier les nombreux praticiens en santé publique qui ont participé aux rencontres, ateliers et webinaires du cercle d'apprentissage, et qui ont révisé plusieurs ébauches au cours du projet.

contre la pauvreté et les travaux en matière de développement durable, qui tous contribueront à faire progresser l'équité en santé.

Bien que l'étape de l'évaluation soit importante dans le cadre d'action que nous présentons, nous n'avons trouvé aucune évaluation décrivant une contribution à l'action spécifique des RESP en matière de déterminants sociaux de la santé. Collectivement, nous devons accroître le corpus de données probantes sur l'évaluation et les rapports, car il s'agit d'une pratique prometteuse en matière de lutte contre les iniquités en santé ${ }^{14}$. Nous proposons ainsi deux domaines de recherche et espérons pouvoir soutenir des travaux concertés sur

\section{Conflits d'intérêts} tion de conflits d'intérêts.

\section{Contributions des auteurs et avis}

LAD a dirigé le projet et a conceptualisé et rédigé la première version du manuscrit. SS, VM, MHB et DA ont fourni la documentation pertinente issue de chaque programme des Centres de collaboration nationale et ont donné leur avis sur les différentes versions du manuscrit. Tous les auteurs ont approuvé la version finale de l'article.
Les auteurs déclarent ne pas être en posi- 
Le contenu de cet article et les opinions qui y sont exprimées n'engagent que les auteurs et ne sont pas forcément représentatifs de la position du Gouvernement du Canada.

\section{Références}

1. Centre de collaboration nationale des déterminants de la santé. Apprenons ensemble : qu'est qu'un rapport sur l'état de santé d'une population et pourquoi est-ce important? Antigonish (N.-É.) : CCNDS, Université St. Francis Xavier; 2012. En ligne à : http://nccdh .ca/images/uploads/Apprenons_ ensemble_3.pdf

2. Braveman PA, Kumanyika S, Fielding $J$, et al. Health, disparities and health equity: the issue is justice. Am J Public Health. 2011;101:S149-S155. doi: 10.2105 /AJPH.2010.300062.

3. Whitehead M, Dahlgren G. Concepts and principles for tackling social inequities in health: levelling up part 1 . Copenhague (Denmark) : World Health Organization; 2006. En ligne à : http:// www.euro.who.int/_data/assets/pdf_ file/0010/74737/E89383.pdf

4. Centre de collaboration nationale des déterminants de la santé. Glossaire français des principaux termes sur l'équité en santé. Antigonish (N.-É.) : CCNDS, Université St. Francis Xavier; 2014. En ligne à : http://nccdh.ca /images/uploads/comments/Glossary _FR.pdf

5. Centre de collaboration nationale des déterminants de la santé. Le rôle de la santé publique dans l'amélioration de l'équité en santé : parlons-en. Antigonish (N.-É.) : CCNDS, Université St. Francis Xavier; 2013. En ligne à : http://nccdh .ca/images/uploads/PHR_FR_Final.pdf

6. Service de santé publique de Sudbury et du district. 10 pratiques prometteuses pour réduire les iniquités sociales en matière de santé dans les cadres locaux de santé publique : document d'information technique. Sudbury (Ont.) : Service de santé publique de Sudbury et du district; 2016. En ligne à : https://www.sdhu.com/wp-content /uploads/2016/05/10_Promising _Practices_Technical_Briefing_FR.pdf
7. Centre de collaboration nationale des déterminants de la santé. L'équité en santé : parlons-en. Antigonish (N.-É.) : CCNDS, Université St. Francis Xavier; 2013. En ligne à : http://nccdh.ca /images/uploads/comments/Lequite_ en_sante_Parlons-en_2013.pdf

8. Agence de la santé publique du Canada. Le rapport de l'administrateur en chef de la santé publique sur l'état de la santé publique au Canada, 2008 : s'attaquer aux inégalités en santé. Ottawa (Ont.) : ASPC; 2008. En ligne à : http://www.phac-aspc.gc.ca/cphorsphc -respcacsp/2008/fr-rc/pdf/CPHO -Report-f.pdf

9. Lemstra M, Neudorf C. Health disparity in Saskatoon: analysis to intervention. Saskatoon (Sask.) : Saskatoon Health Region; 2014. En ligne à : http://www.caledoninst.org/Special \% 20Projects/CG-COP/Docs/Health DisparityRept-complete.pdf

10. Service de santé publique de Sudbury et du district. Possibilités pour tous : le chemin vers l'équité en matière de santé. Sudbury (Ont.) : Service de santé publique de Sudbury et du district; 2013. En ligne à : https://www .sdhu.com/wp-content/uploads/2015 /10/Opportunity-for-All-Report_FINAL_ for-web_FR_FileInfo.pdf

11. Toronto Public Health. The unequal city 2015: Income and health inequities in Toronto: technical report. Toronto (Ont.) : Toronto Public Health; 2015. En ligne à : http://www1.toronto .ca/City \% 200f\% 20Toronto/Toronto $\%$ 20Public \% 20Health/Performance $\% 20 \& \% 20$ Standards / Health \% 20 Surveillance \%20and\%20Epidemiology /Files/pdf/Technical \% 20Report \% 20 FINAL \%20PRINT_AODA.pdf

12. Centres de collaboration nationale en santé publique et Centre de collaboration nationale des déterminants de la santé. Cadre d'action axé sur l'équité pour l'élaboration de rapports sur l'état de santé des populations. Antigonish (N.-É.) : CCNDS, Université St. Francis Xavier; 2016. En ligne à : http://nccph.ca/images/uploads/general /PHSR_Action_Framework_FR_final .pdf
13. Centres de collaboration nationale en santé publique. À propos [Internet]. CCNSP; [cité le 31 juil. 2017]. En ligne à la page : http://ccnsp.ca/about-us/

14. Centre de collaboration nationale des déterminants de la santé. Apprenons ensemble : qu'avons-nous appris sur l'intégration de la notion d'équité dans les rapports sur l'état de santé des populations? Comment avons-nous fait notre apprentissage? Antigonish (N.-É.) : CCNDS, Université St. Francis Xavier; 2013. En ligne à : http://nccdh .ca/images/uploads/Equity_Integration _FR_Final_Fr.pdf

15. Marchildon GP. Canada : examen du système de santé 2013 [Internet]. Copenhague, Observatoire européen des systèmes et des politiques de santé; 2013 [cité le 31 juil. 2017]. En ligne à : http://www.euro.who.int /en/about-us/partners/observatory /publications/health-system-reviews -hits/full-list-of-country-hits/canada -hit-2013

16. Réseau pancanadien de santé publique. Plan directeur pour un système fédéré de surveillance de la santé publique au Canada [Internet]. Ottawa (Ont.) : Réseau pancanadien de santé publique; 2016 [cité le 31 juil. 2017]. En ligne à : http://www.phn-rsp.ca/pubs/bfsph -psfsp-2016/index-fra.php

17. Centre de collaboration nationale sur les politiques publiques et la santé. Profil structurel de la santé publique au Canada [Internet]. Montréal (Qué.) : CCNPPS; [cité le 27 juil. 2017]. En ligne à : http://www.ccnpps.ca/712 /profil-structurel-de-la-sante-publique -au-canada.ccnpps

18. Hall HI, Correa A, Yoon PW, Braden CR. Lexicon, definitions, and conceptual framework for public health surveillance. MMWR Surveill Summ. 2012; 61(Suppl):10-14.

19. Fawcett S, Holt C, Schultz J. Some recommended practice areas for community health improvement (report to the CDC, office of prevention through healthcare). Kansas : Work Group for Community Health and Development, University of Kansas; 2011. En ligne à : http://ctb.ku.edu/sites/default/files /site_files/recommended_practices_ for_enhancing_community_health_ improvement.pdf 
20. Association for Community Health Improvement. ACHI community health assessment tool kit [Internet]. Health Research and Educational Trust; 2007 [cité le 31 juil. 2017]. En ligne à : http://www.assesstoolkit.org/

21. Centre de collaboration nationale des déterminants de la santé. Apprenons ensemble : examen des données probantes concernant l'objectif des rapports sur l'état de santé des populations [Internet]. Antigonish (N.-É.) : CCNDS, Université St. Francis Xavier; 2012. En ligne à : http://nccdh.ca/images/uploads /Apprenons_ensemble_4.pdf

22. Bonnefoy J, Morgan A, Kelly MP, Butt J, Bergman V. Constructing the evidence base on the social determinants of health: a guide. Measurement and Evidence Knowledge Network (MEKN) of the WHO Commission on Social Determinants of Health; 2007. En ligne à : www.who.int/social_determinants /knowledge_networks/add_documents /mekn_final_guide_112007.pdf

23. Allan J, McPherson N, Williams C, et al. Prairie mountain health community health assessment 2015. Souris (Man.) : Prairie Mountain Health; 2015. En ligne à : http://www.prairiemountainhealth .ca/images/Community_Health_ Assessment.pdf

24. Winnipeg Regional Health Authority. Health for all: building Winnipeg's health equity action plan [Internet]. Winnipeg (Man.) : Winnipeg Regional Health Authority; 2013. En ligne à : http: //www.wrha.mb.ca/about/healthequity /files/HealthForAll_Documentwith links.pdf

25. Vancouver Coastal Health. Reducing health disparities in Vancouver Coastal Health communities: population health priorities [Internet]. Vancouver (C.-B.): Vancouver Coastal Health; 2008.

26. Centre for Healthcare Innovation and Winnipeg Regional Health Authority. 2014 community health assessment [Internet]. Winnipeg (Man.): WRHA \& CHI Evaluation Platform; 2015 [cité le 31 juil. 2017]. En ligne à : http://www .wrha.mb.ca/research/cha2014/index .php
27. Provincial Health Services Authority. Development of priority health equity indicators for British Columbia: process and outcome report. Vancouver (C.-B.): Provincial Health Services Authority, Population and Public Health Program; 2014. En ligne à : http://www.phsa.ca /Documents/developmentofpriority healthequityindicatorsreportw.pdf

28. Réseau pancanadien de santé publique. Indicateurs des disparités sur le plan de la santé. Un rapport du Groupe d'experts de la promotion de la santé de la population et du Groupe des modes de vie sains pour le Réseau pancanadien de santé publique. Réseau pancanadien de santé publique; 2010. En ligne à : http:// www.phn-rsp.ca/pubs/ihi-idps/pdf /Indicators-of-Health-Inequalities -Report-PHPEG-Feb-2010-FR.pdf

29. Bell N, Hayes MV. The Vancouver area neighbourhood deprivation index (vandix): a census-based tool for assessing small-area variations in health status. Can J Public Health. 2012;103(Suppl. 2): S32.

30. Chateau D, Metge C, Prior H, Soodeen $\mathrm{R}$. Learning from the census: the socioeconomic factor index (SEFI) and health outcomes in Manitoba. Can J Public Health. 2012;103(Suppl 2):S27.

31. Pampalon R, Hamel D, Gamache P, Philibert MD, Raymond G, Simpson A. An area-based material and social deprivation index for public health in Québec and Canada. Can J Public Health. 2012;103(Suppl 2):S22.

32. Institut canadien d'information sur la santé (ICIS). Tendances des inégalités en santé liées au revenu au Canada : rapport technique. Ottawa (Ont.) : ICIS; 2015. En ligne à : https://secure.cihi .ca/free_products/trends_in_income _related_inequalities_in_canada_2015 _fr.pdf

33. Norheim OF. Gini impact analysis: measuring pure health inequity before and after interventions. Public Health Ethics. 2010;3(3):282-292. doi: 10.1093 /phe/phq017.

34. Harper S, King NB, Meersman SC, Reichman ME, Breen N, Lynch J. Implicit value judgments in the measurement of health inequalities. Milbank Q. 2010;88(1):4-29. doi: 10.1111/j.1468 $-0009.2010 .00587 . x$.
35. Asada Y, Hurley J, Norheim OF, Johri M. A three-stage approach to measuring health inequalities and inequities. Int J Equity Health. 2014;13:98. doi: 10.1186/s12939-014-0098-y.

36. Moonesinghe R, Bouye K, PenmanAguilar A. Difference in health inequity between two population groups due to a social determinant of health. Int $\mathrm{J}$ Environ Res Public Health. 2014;11(12):13083. doi: 10.3390 /ijerph111213074.

37. Gakidou E, Fullman N. Monitoring health inequalities: measurement considerations and implications [Internet]. Brisbane (AUS) : Health Information Systems Knowledge Hub, School of Population Health, University of Queensland; 2012 [cité le 31 juil. 2017]. En ligne à : http://www.healthdata .org/research-article/monitoring -health-inequalities-measurement -considerations-and-implications

38. Corburn J, Cohen AK. Why we need urban health equity indicators: integrating science, policy, and community. PLoS Med. 2012;9(8):e1001285. doi: 10.1371/journal.pmed.1001285.

39. Freemantle J, Ring I, Arambula Solomon TG, et al. Indigenous mortality (revealed): the invisible illuminated. Am J Public Health. 2015;105(4): 644-652. doi: 10.2105/AJPH.2014.301994.

40. Adelson N. The embodiment of inequity: health disparities in aboriginal Canada. Can J Public Health. 2005; 6(Suppl 2):S61.

41. King M, Smith A, Gracey M. Indigenous health part 2: the underlying causes of the health gap. Lancet. 2009;374(9683): 76-85. doi: 10.1016/S0140-6736(09) 60827-8.

42. Centre de gouvernance de l'information des Premières Nations. L'Enquête régionale sur la santé des Premières Nations (ERS) 2008/10 : rapport national sur les adultes, les adolescents et les enfants qui vivent dans les communautés des Premières Nations [Internet]. Ottawa (Ont.) : CGIPN; 2012. En ligne à : http://fnigc.ca/sites/default/files /docs/rhs_phase_2_2008_2010_fr_ final_0.pdf 
43. Brown C, Harrison D, Burns H, Ziglio E. Governance for health equity: taking forward the equity values and goals of health 2020 in the WHO European region [Internet]. Copenhague (Danemark): World Health Organization Regional Office for Europe; 2013. En ligne à : http://www.euro.who.int /_data/assets/pdf_file/0020/235712 /e96954.pdf

44. Centre de collaboration nationale des méthodes et outils. Santé publique fondée sur les données probantes [Internet]. Hamilton (Ont.) : CCNMO, Université McMaster; 2016 [cité le 31 juil. 2017]. En ligne à : http://www .nccmt.ca/fr/au-sujet-du-ccnmo/eiph

45. Robert Wood Johnson Foundation. County health rankings and roadmaps - action center [Internet]. Robert Wood Johnson Foundation; [cité le 31 juil. 2017]. Consultable en ligne à la page : http://www.countyhealthrankings.org /roadmaps/action-center

46. Graham H. Health inequalities, social determinants and public health policy. Policy Polit. 2009;37(4):463-479. doi: 10.1332/030557309X445618.

47. Embrett MG, Randall GE. Social determinants of health and health equity policy research: exploring the use, misuse, and non-use of policy analysis theory. Soc Sci Med. 2014;108:147155. doi: 10.1016/j.socscimed.2014.03 .004 .

48. Nakaima A, Sridharan S, Gardner B. Towards a performance measurement system for health equity in a local health integration network. Eval Program Plann. 2013;36(1):204-212. doi: 10.1016/j.evalprogplan.2012.03.009.

49. Centres de collaboration nationale en santé publique et Centre de collaboration nationale des déterminants de la santé. Sommaire - Élaboration de rapports sur l'état de santé des populations en tenant compte de l'équité : cadre d'action [Internet]. Antigonish (N.-É.) : CCNDS; 2016. En ligne à : http://nccdh.ca/images/uploads /comments/PHSR_Action_Framework _Summary_FR_June_15.pdf 